\title{
Laktoseintoleranz: kein Verlass auf subjektive Beschwerden
}

Die Diagnose Laktoseintoleranz wird meist aufgrund anamnestischer Angaben gestellt, d.h. aus dem zeitlichen Zusammenhang zwischen Laktosegenuss und dem Auftreten von Beschwerden. Doch diese Vorgehensweise ist nicht zuverlässig.

\begin{abstract}
A ngaben zu subjektiven Beschwerden erlauben keine zuverlässige Diagnose einer Laktoseintoleranz. Darauf deuten die Ergebnisse einer prospektiven Studie mit 353 Patienten hin, bei denen zur Abklärung zusätzlich ein Laktose- $\mathrm{H}_{2}$-Atemtest durchgeführt wurde. Zuerst mussten die Patienten einen validierten Fragebogen zu den Symptomen beantworten, die nach dem Verzehr von Milchprodukten im Alltag auftraten. Nach Durchführung des Atemtests wurde dann die durch die Laktosegabe induzierte Symptomatik dokumentiert.

Bei 164 Patienten wurde anhand des Laktose- $\mathrm{H}_{2}$-Atemtests eine Laktose-
\end{abstract}

malabsorption diagnostiziert, bei den übrigen wurde eine solche ausgeschlossen. Der Symptomscore im Alltag war jedoch in beiden Gruppen ähnlich. Allerdings war der Symptomscore bei Patienten ohne Laktosemalabsorption im Alltag viermal höher als der Symptomscore während der Durchführung des Laktose- $\mathrm{H}_{2}$-Atemtests. Nur bei den Patienten mit nachgewiesener Laktosemalabsorption war die Intensität der Symptome im Alltag und während der Laktosebelastung vergleichbar.

Fazit: Bei Verdacht auf eine Laktoseintoleranz sollte immer eine Objektivie-

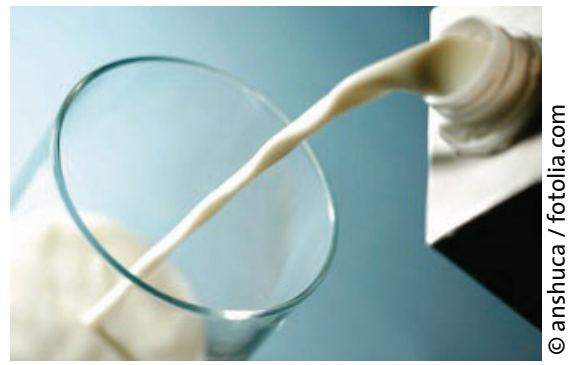

Nicht immer ist Laktose der Grund dafür, wenn Milch nicht verteragen wird.

rung der Diagnose mittels Atemtest erfolgen. Der Nachweis oder Ausschluss einer Laktoseintoleranz ist wichtig, auch um die Patienten vor unnötigen endoskopischen Abklärungen im Hinblick auf das Vorliegen einer chronisch entzündlichen Darmerkrankung oder der Fehldiagnose eines Reizdarmsyndroms zu bewahren. Dr. Peter Stiefelhagen

Casellas F et al. Subjective perception of lactose intolerance does not always indicate lactose malabsorption. Clin Gastroenterol Hepatol 2010; 8: 581-6 